\title{
ON THE DYNAMICS OF A QUADRATIC SCHRÖDINGER SYSTEM IN DIMENSION $n=5$
}

\author{
NORMAN NOGUERA AND ADEMIR PASTOR
}

\begin{abstract}
In this work we give a sharp criterion for the global well-posedness, in the energy space, for a system of nonlinear Schrödinger equations with quadratic interaction in dimension $n=5$. The criterion is given in terms of the charge and energy of the ground states associated with the system, which are obtained by minimizing a Weinstein-type functional. The main result is then obtained in view of a sharp Gagliardo-Nirenberg-type inequality.
\end{abstract}

\section{INTRODUCTION}

In this notes we are interested in the following initial-value problem

$$
\left\{\begin{array}{l}
i \partial_{t} u+\frac{1}{2 m} \Delta u=\lambda \bar{u} v \\
i \partial_{t} v+\frac{1}{2 M} \Delta v=\mu u^{2}, \\
(u(x, 0), v(x, 0))=\left(u_{0}, v_{0}\right) .
\end{array}\right.
$$

where $u, v: \mathbb{R}^{n} \times \mathbb{R} \rightarrow \mathbb{C},(x, t) \in \mathbb{R}^{n} \times \mathbb{R}, \Delta$ is the Laplacian operator, $m, M>0$ are real constants and $\lambda, \mu \in \mathbb{C}$. System (1.1) can be regarded as a non-relativistic limit of a KleinGordon system under the so called mass resonance condition $M=2 m$. Also, similar systems appear as models for the interaction of waves propagating in a $\chi^{(2)}$ dispersive media (see, for instance, [3]). From the mathematical point of view, the study of nonlinear Schrödinger systems with quadratic interaction has been increasing in recent years. To cite a few, we refer the reader to [3], 44, [7, [8], [9], [12, [14, [15], [20, and references therein.

An almost complete study of system (1.1) in $L^{2}\left(\mathbb{R}^{n}\right)$ and $H^{1}\left(\mathbb{R}^{n}\right)$ was undertaken in [9] (see also [7], 8] ). First of all, one should note that $L^{2}\left(\mathbb{R}^{n}\right)$ and $H^{1}\left(\mathbb{R}^{n}\right)$ are critical spaces (in the sense of scaling) for $n=4$ and $n=6$. In particular, it has been shown the local well-posedness in $L^{2}\left(\mathbb{R}^{n}\right), 1 \leq n \leq 4$ and in $H^{1}\left(\mathbb{R}^{n}\right), 1 \leq n \leq 6$. The method used to prove these results was the contraction argument combined with the well known Strichartz estimates (see, for instance, [2] or [16]). Under the assumption that there exists $c \in \mathbb{R} \backslash\{0\}$ such that $\lambda=c \bar{\mu}$ the global well-posedness in $L^{2}\left(\mathbb{R}^{n}\right), 1 \leq n \leq 3$ and in $H^{1}\left(\mathbb{R}^{n}\right), 1 \leq n \leq 3$, were also established. This condition on the parameters is necessary in order to obtain the conservation of the charge and the energy, which in turn imply a priori estimates. Since $L^{2}\left(\mathbb{R}^{4}\right)$ is a critical space, the global existence for $n=4$ requires an additional assumption on the initial data. To be more precise on the results, here and throughout the paper we assume:

There exist a constant $c \in \mathbb{R} \backslash\{0\}$ such that $\lambda=c \bar{\mu}$.

By introducing the change of variables

$$
\tilde{u}(x, t)=\sqrt{\frac{c}{2}}|\mu| u\left(\sqrt{\frac{1}{2 m}} x, t\right), \quad \tilde{v}(x, t)=-\frac{\lambda}{2} v\left(\sqrt{\frac{1}{2 m}} x, t\right),
$$

2010 Mathematics Subject Classification. 35Q55, 35B44, 35J47, 35A01.

Key words and phrases. Global well-posedness; Blow up; Ground states solutions. 
we deduce that, after dropping the tildes, (1.1) is equivalent to

$$
\left\{\begin{array}{l}
i \partial_{t} u+\Delta u=-2 v \bar{u}, \\
i \partial_{t} v+\kappa \Delta v=-u^{2}, \\
(u(x, 0), v(x, 0))=\left(u_{0}, v_{0}\right),
\end{array}\right.
$$

with $\kappa=m / M$. So in what follows we will be concerned with system (1.4) instead of (1.1).

It is not difficult to see that (1.4) conserves the charge and the energy, which are given, respectively, by

$$
Q(u(t), v(t))=\|u(t)\|_{L^{2}}^{2}+2\|v(t)\|_{L^{2}}^{2}
$$

and

$$
E(u(t), v(t))=\|\nabla u(t)\|_{L^{2}}^{2}+\kappa\|\nabla v(t)\|_{L^{2}}^{2}-2 \operatorname{Re}\left(v(t), u^{2}(t)\right)_{L^{2}} .
$$

With this terminology in hand it was established in 9 , that if $Q\left(u_{0}, v_{0}\right)<Q\left(\phi_{0}, \psi_{0}\right)$, where $\left(\phi_{0}, \psi_{0}\right)$ is any ground state associated with (1.4) (see Section 2 for details) then the corresponding solution is global in $H^{1}\left(\mathbb{R}^{4}\right)$. This result is similar to the classical one established by Weinstein 22 for the mass-critical NLS equation. To prove this result it was necessary to show a vectorial Gagliardo-Nirenberg-type inequality. In addition, under the mass resonance condition $\kappa=1 / 2$ is was shown that this result is sharp in the sense that there exists a particular initial data $\left(u_{0}^{*}, v_{0}^{*}\right)$ such that $Q\left(u_{0}^{*}, v_{0}^{*}\right)=Q\left(\phi_{0}, \psi_{0}\right)$ but the corresponding local solution blows up in finite time. It is to be observed that the existence of ground state solutions was established in any dimension $1 \leq n \leq 5$. The used methods were based on the concentration-compactness argument in dimension $n=1$ and on the Strauss' compactness lemma in dimension $2 \leq n \leq 5$ (see [21]).

We point out that, still under the condition $\kappa=1 / 2$, if $E\left(u_{0}, v_{0}\right)<0$ (or $E\left(u_{0}, v_{0}\right)=0$ and $\left(u_{0}, v_{0}\right)$ has negative momentum) then the local solution also blows up in finite time in dimension $4 \leq n \leq 6$ (see Theorem 3.12 in [9]).

At this point we observe that in [9] nothing was said concerning the global well-posedness in $H^{1}\left(\mathbb{R}^{5}\right)$. To the best of our knowledge there are no results in this direction in the current literature. So, our main purpose in this notes is to obtain a sufficient sharp condition for global well-posedness in $H^{1}\left(\mathbb{R}^{5}\right)$; this is the $L^{2}$ supercritical and $H^{1}$ subcritical case.

Before stating our results, let us introduce the functional

$$
K(u, v)=\|\nabla u\|_{L^{2}}^{2}+\kappa\|\nabla v\|_{L^{2}}^{2} .
$$

Our main theorems reads as follows.

Theorem 1.1 (Sufficient condition for global existence). Assume $\left(u_{0}, v_{0}\right) \in H^{1}\left(\mathbb{R}^{5}\right) \times H^{1}\left(\mathbb{R}^{5}\right)$ and let $(u(t), v(t))$ be the corresponding solution of (1.4), defined in the maximal interval of existence, say, I. Assume that

$$
E\left(u_{0}, v_{0}\right) Q\left(u_{0}, v_{0}\right)<E(\phi, \psi) Q(\phi, \psi) .
$$

If

$$
K\left(u_{0}, v_{0}\right) Q\left(u_{0}, v_{0}\right)<K(\phi, \psi) Q(\phi, \psi),
$$

then

$$
K(u(t), v(t)) Q\left(u_{0}, v_{0}\right)<K(\phi, \psi) Q(\phi, \psi), \quad \forall t \in I .
$$

In particular the initial-value problem (1.4) is globally well-posed in $H^{1}\left(\mathbb{R}^{5}\right) \times H^{1}\left(\mathbb{R}^{5}\right)$.

Here, $(\phi, \psi)$ is any ground state solution for (2.1) with $\omega=1$ (see Section 2). Under the mass resonance condition $\kappa=1 / 2$, next theorem shows that (1.9) is a sharp condition for the global existence.

Theorem 1.2 (Existence of blow-up solutions). Let $(\phi, \psi)$ be a ground state solution for (2.1) with $\omega=1$. Assume $\kappa=1 / 2$ and $\left(u_{0}, v_{0}\right) \in H^{1}\left(\mathbb{R}^{5}\right) \times H^{1}\left(\mathbb{R}^{5}\right)$ and let $I$ be the maximal time interval of existence of the solution $(u(t), v(t))$. Suppose

$$
E\left(u_{0}, v_{0}\right) Q\left(u_{0}, v_{0}\right)<E(\phi, \psi) Q(\phi, \psi),
$$


and

$$
K\left(u_{0}, v_{0}\right) Q\left(u_{0}, v_{0}\right)>K(\phi, \psi) Q(\phi, \psi) .
$$

If $\left(x u_{0}, x v_{0}\right) \in L^{2}\left(\mathbb{R}^{5}\right) \times L^{2}\left(\mathbb{R}^{5}\right)$ or $u_{0}, v_{0}$ are radial functions, then $I$ is finite, which means to say that $(u(t), v(t))$ blows up in finite time.

Theorems 1.1 and 1.2 are inspired in [10, where similar results were obtained for the classical Schrödinger equation.

This paper is organized as follows. In section 2 we state preliminary results concerned with the ground state solutions. In particular, their existence is shown via minimization of Weinstein's functional. We also establish a sharp Gagliardo-Nirenberg inequality. In section 3. we prove Theorems 1.1 and 1.2

\section{GROUND STATES AND THEIR PROPERTIES}

In this section we will introduce the main tools to prove Theorems 1.1 and 1.2. In general, we use the standard notation in the theory of partial differential equations.

2.1. Preliminary results. Let us start by introducing the notion of ground states. First of all, we recall that a standing wave solution for (1.4) is a solution of the form

$$
(u, v)=\left(e^{i \omega t} \phi(x), e^{2 i \omega t} \psi(x)\right),
$$

where $\omega>0$ is a real parameter and $\phi, \psi$ are real-valued functions, which may depend on $\omega$, with a suitable decay at infinity. By replacing this ansatz in (1.4), we face the elliptic system

$$
\left\{\begin{array}{l}
-\Delta \phi+\omega \phi=2 \phi \psi, \\
-\kappa \Delta \psi+2 \omega \psi=\phi^{2}
\end{array}\right.
$$

where as before $\kappa=m / M$. In what follows, $\kappa$ will be thought as any positive real constant.

Definition 2.1. A pair $(\phi, \psi) \in H^{1}\left(\mathbb{R}^{n}\right) \times H^{1}\left(\mathbb{R}^{n}\right)$ is called a solution (or a weak solution) of (2.1) if

$$
\begin{aligned}
& \int(\nabla \phi \cdot \nabla f+\omega \phi f) d x=2 \int \phi \psi f d x, \\
& \int(\kappa \nabla \psi \cdot \nabla g+2 \omega \psi g) d x=\int \phi^{2} g d x,
\end{aligned}
$$

for any $f, g \in C_{0}^{\infty}\left(\mathbb{R}^{n}\right)$.

As usual, from the standard elliptic regularity theory (see, for instance, [2] or [6]), weak solutions are indeed smooth and satisfy (2.1) in the usual sense. The so called ground state solutions are usually obtained as minimizers of some functional connecting its critical points with the solutions of (2.1). The functional of interest here is defined by

$$
\begin{aligned}
I_{\omega}(\phi, \psi) & =\frac{1}{2}\left(\|\nabla \phi\|_{L^{2}}^{2}+\kappa\|\nabla \psi\|_{L^{2}}^{2}\right)+\frac{\omega}{2}\left(\|\phi\|_{L^{2}}^{2}+2\|\psi\|_{L^{2}}^{2}\right)-\int \phi^{2} \psi d x \\
& \equiv \frac{1}{2} E(\phi, \psi)+\frac{\omega}{2} Q(\phi, \psi) .
\end{aligned}
$$

In particular, it is easily seen that $(\phi, \psi)$ is a solution of (2.1) if and only if it is a critical point of $I_{\omega}$. As we said, among all solutions of (1.4) stand out the so called ground states we shall define next.

Definition 2.2. A solution $\left(\phi_{0}, \psi_{0}\right) \in H^{1}\left(\mathbb{R}^{n}\right) \times H^{1}\left(\mathbb{R}^{n}\right)$ of (2.1) is called a ground state if

$$
I_{\omega}\left(\phi_{0}, \psi_{0}\right)=\inf \left\{I_{\omega}(\phi, \psi) ;(\phi, \psi) \in \mathcal{C}_{\omega}\right\},
$$

where

$$
\mathcal{C}_{\omega}:=\left\{(\phi, \psi) \in H^{1}\left(\mathbb{R}^{n}\right) \times H^{1}\left(\mathbb{R}^{n}\right) ;(\phi, \psi) \text { is a nontrivial critical point of } I_{\omega}\right\} .
$$

The set of ground states for (2.1) will be denoted by $\mathcal{G}_{\omega}$. 
The existence of ground states for (2.1), in dimension $1 \leq n \leq 5$, was already obtained in [9] by minimizing the functional

$$
R_{\omega}(\phi, \psi)=\frac{K(\phi, \psi)+\omega Q(\phi, \psi)}{P(\phi, \psi)^{2 / 3}}
$$

on $\mathcal{P}$, where

$$
P(\phi, \psi):=\int \phi^{2} \psi d x
$$

and

$$
\mathcal{P}:=\left\{(\phi, \psi) \in H^{1}\left(\mathbb{R}^{n}\right) \times H^{1}\left(\mathbb{R}^{n}\right) \backslash\{(0,0)\} ; \int \phi^{2} \psi d x>0\right\} .
$$

However, here we will present a slight different proof based only on Strauss' compactness lemma. Indeed, in [9] the authors used the compactness of the embedding $H_{r}^{1}\left(\mathbb{R}^{n}\right) \hookrightarrow L^{3}\left(\mathbb{R}^{n}\right)$, $2 \leq n \leq 5$ to obtain that any minimizing sequence converges to a ground state (up to scaling). Here, $H_{r}^{1}$ denotes the space of radially symmetric $H^{1}$ functions. Due to the lack of the above mentioned compactness in dimension $n=1$, they employed a concentration-compactness argument on the functional $I_{\omega}=\frac{1}{2}(K+\omega Q)-P$ to obtain the ground states in this case.

In our approach, the ground states will be obtained as minimizers of a Weinstein-type functional. The advantage in using this approach is that, as a byproduct, it also yields the best constant in a vectorial Gagliardo-Nirenberg inequality (see Corollary 2.10 below). This method was used in [9] only for $n=4$.

Although we are mainly concerned with dimension $n=5$, we prove the existence of ground states for $1 \leq n \leq 5$, because it does not demand extra efforts. In fact, instead of using two different approaches for $n=1$ and $2 \leq n \leq 5$, we use the compactness of the embedding $H_{r d}^{1}\left(\mathbb{R}^{n}\right) \hookrightarrow L^{3}\left(\mathbb{R}^{n}\right)$, which holds in any dimension $1 \leq n \leq 5$ (see, for instance, 2, Proposition 1.7.1]). In particular, this simplifies the proof of the existence of ground states in dimension $n=1$. Here $H_{r d}^{1}$ denotes the space of radially symmetric and nonincreasing $H^{1}$ functions.

We start with the following properties.

Lemma 2.1. Let $(\phi, \psi)$ be a solution of (2.1). Then,

$$
\begin{gathered}
P(\phi, \psi)=2 I_{\omega}(\phi, \psi), \\
K(\phi, \psi)=n I_{\omega}(\phi, \psi), \\
\omega Q(\phi, \psi)=(6-n) I_{\omega}(\phi, \psi) .
\end{gathered}
$$

Proof. See Theorem 4.1 in $[9]$.

Remark 2.2. In view of Lemma 2.1] some simple remarks are in order:

(1) From (2.5) we conclude that a solution $(\phi, \psi)$ of (2.1) is a ground state if and only if the charge $Q(\phi, \psi)$ is minimal.

(2) Ground states do not exist if $(6-n) \omega \leq 0$. So, since we are assuming $\omega>0$, ground states do not exist if $n \geq 6$.

(3) If $(\phi, \psi)$ is a solution of (2.1) then (2.3) and 2.4) imply that $P(\phi, \psi)>0$; this means that $\mathcal{C}_{\omega} \subset \mathcal{P}$.

Next we introduce the Weinstein functional

$$
J(\phi, \psi)=\frac{\left(\|\phi\|_{L^{2}}^{2}+2\|\psi\|_{L^{2}}^{2}\right)^{\frac{3}{2}-\frac{n}{4}}\left(\|\nabla \phi\|_{L^{2}}^{2}+\kappa\|\nabla \psi\|_{L^{2}}^{2}\right)^{\frac{n}{4}}}{\int \phi^{2} \psi d x} \equiv \frac{Q(\phi, \psi)^{\frac{3}{2}-\frac{n}{4}} K(\phi, \psi)^{\frac{n}{4}}}{P(\phi, \psi)} .
$$

Note that if $(\phi, \psi)$ is a solution of (2.1) then, in view of Lemma 2.1 and Remark 2.2, $J(\phi, \psi)$ is well-defined. In the following, we are going to use Lemma 2.1 in order to get a relation between functionals $J$ and $I_{\omega}$. More precisely, we have. 
Lemma 2.3. Let $(\phi, \psi)$ be a nontrivial solution of (2.1) then

$$
J(\phi, \psi)=\frac{n^{\frac{n}{4}}}{2}\left(\frac{6-n}{\omega}\right)^{\frac{3}{2}-\frac{n}{4}} I_{\omega}(\phi, \psi)^{\frac{1}{2}} .
$$

Proof. Combining expressions (2.3), (2.4) and (2.5) in Lemma 2.1 and the definition of $J$, we have

$$
\begin{aligned}
J(\phi, \psi) & =\frac{Q(\phi, \psi)^{\frac{3}{2}-\frac{n}{4}} K(\phi, \psi)^{\frac{n}{4}}}{P(\phi, \psi)} \\
& =\frac{\left(\frac{6-n}{\omega}\right)^{\frac{3}{2}-\frac{n}{4}} I_{\omega}(\phi, \psi)^{\frac{3}{2}-\frac{n}{4}} n^{\frac{n}{4}} I_{\omega}(\phi, \psi)^{\frac{n}{4}}}{2 I_{\omega}(\phi, \psi)} \\
& =\frac{n^{\frac{n}{4}}}{2}\left(\frac{6-n}{\omega}\right)^{\frac{3}{2}-\frac{n}{4}} I_{\omega}(\phi, \psi)^{\frac{1}{2}}
\end{aligned}
$$

The proof of the lemma is thus completed.

As an immediate consequence, we obtain the following.

Corollary 2.4. A nontrivial solution $(\phi, \psi)$ of (2.1) is a ground state if and only if it is a minimizer of $J$.

In view of Corollary 2.4, the idea to obtain the ground states is to minimize $J$ on the set $\mathcal{P}$.

2.2. Existence of ground states. Before proceeding we note that if we know a ground state for $\omega=1$ then we know a ground state for any $\omega>0$ (see Proposition 4.3 in [9]). In fact, if $\left(\phi_{1}, \psi_{1}\right)$ is a ground state for (2.1) with $\omega=1$, then

$$
\left(\phi_{\omega}(x), \psi_{\omega}(x)\right)=\left(\omega \phi_{1}(\sqrt{\omega} x), \omega \psi_{1}(\sqrt{\omega} x)\right),
$$

is a ground state for (2.1), with $\omega>0$.

Our main theorem concerning ground states is the following.

Theorem 2.5 (Existence of ground states). For $1 \leq n \leq 5$, the infimum

$$
\alpha_{1}=\inf _{(\phi, \psi) \in \mathcal{P}} J(\phi, \psi)
$$

is attained at a pair the functions $\left(\phi_{0}, \psi_{0}\right) \in \mathcal{P}$ such that

(i) $\phi_{0}$ and $\psi_{0}$ are non-negative and radially symmetric;

(ii) There exist $t_{0}>0$ and $l_{0}>0$ such that $(\phi, \psi)=\left(t_{0} \delta_{l_{0}} \phi_{0}, t_{0} \delta_{l_{0}} \psi_{0}\right)$ is a positive ground state of (2.1) with $\omega=1$, where $\left(\delta_{l} f\right)(x)=f(x / l)$;

(iii) If $(\tilde{\phi}, \tilde{\psi})$ is any ground state of (2.1), with $\omega=1$, then

$$
\alpha_{1}=\frac{n^{\frac{n}{4}}}{2}(6-n)^{1-\frac{n}{4}} Q(\tilde{\phi}, \tilde{\psi})^{1 / 2} .
$$

Remark 2.6. If $n=4$ then the constant $\alpha_{1}$ in (2.9) reduces to $\alpha_{1}=2 Q(\tilde{\phi}, \tilde{\psi})^{1 / 2}$, which is exactly the one obtained in [9, Theorem 5.1].

Below we will prove Theorem 2.5. To begin with, we show the following.

Lemma 2.7. If $\alpha_{1}$ is defined as in (2.8) then $\alpha_{1}>0$.

Proof. First we recall the Gagliardo-Nirenberg inequality $\|\phi\|_{L^{3}} \leq C\|\nabla \phi\|_{L^{2}}^{\frac{n}{6}}\|\phi\|_{L^{2}}^{1-\frac{n}{6}}$. Thus, from Hölder's inequality,

$$
\begin{aligned}
P(\phi, \psi) & \leq\|\phi\|_{L^{3}}^{2}\|\psi\|_{L^{3}} \\
& \leq C^{3}\left(\|\nabla \phi\|_{L^{2}}^{\frac{n}{6}}\|\phi\|_{L^{2}}^{1-\frac{n}{6}}\right)^{2}\|\nabla \psi\|_{L^{2}}^{\frac{n}{6}}\|\psi\|_{L^{2}}^{1-\frac{n}{6}} \\
& =C^{3} \kappa^{-\frac{n}{12}} 2^{\frac{n}{12}-\frac{1}{2}} Q(\phi, \psi)^{\frac{3}{2}-\frac{n}{4}} K(\phi, \psi)^{\frac{n}{4}} .
\end{aligned}
$$


Then, from the definition of $\alpha_{1}$,

$$
0<C^{-3} \kappa^{\frac{n}{12}} 2^{-\frac{n}{12}+\frac{1}{2}} \leq \alpha_{1}
$$

which yields the desired.

In the sequel, given any non-negative function $\phi \in H^{1}\left(\mathbb{R}^{n}\right)$ we denote by $\phi^{*}$ its symmetricdecreasing rearrangement (see, for instance, [13]). Also, for any $l>0,\left(\delta_{l} f\right)(x)=f(x / l)$.

Lemma 2.8. Assume $a, l>0$ and $(\phi, \psi) \in H^{1}\left(\mathbb{R}^{n}\right) \times H^{1}\left(\mathbb{R}^{n}\right)$. Then the following properties hold:

(i) $Q\left(a \delta_{l} \phi, a \delta_{l} \psi\right)=a^{2} l^{n} Q(\phi, \psi), \quad Q^{\prime}\left(a \delta_{l} \phi, a \delta_{l} \psi\right)(u, v)=a l^{n} Q^{\prime}(\phi, \psi)\left(\delta_{l^{-1}} u, \delta_{l^{-1}} v\right)$;

(ii) $K\left(a \delta_{l} \phi, a \delta_{l} \psi\right)=a^{2} l^{n-2} K(\phi, \psi), \quad K^{\prime}\left(a \delta_{l} \phi, a \delta_{l} \psi\right)(u, v)=a l^{n-2} K^{\prime}(\phi, \psi)\left(\delta_{l^{-1}} u, \delta_{l^{-1}} v\right)$;

(iii) $P\left(a \delta_{l} \phi, a \delta_{l} \psi\right)=a^{3} l^{n} P(\phi, \psi), \quad P^{\prime}\left(a \delta_{l} \phi, a \delta_{l} \psi\right)(u, v)=a^{2} l^{n} P^{\prime}(\phi, \psi)\left(\delta_{l^{-1}} u, \delta_{l^{-1}} v\right)$. In addition, if $\phi$ and $\psi$ are non-negative then

(iv) $Q\left(\phi^{*}, \psi^{*}\right)=Q(\phi, \psi)$;

(v) $K\left(\phi^{*}, \psi^{*}\right) \leq K(\phi, \psi)$;

(vi) $P\left(\phi^{*}, \psi^{*}\right) \geq P(\phi, \psi)$.

Proof. The proofs are simple calculations. For parts (iv), (v), and (vi) see, for instance, Chapters 6 and 16 in [13].

The following lemma establishes some properties of the functional $J$, under the transformations of scaling, dilation, and symmetrization.

Lemma 2.9. If $a, l>0$ and $(\phi, \psi) \in \mathcal{P}$, then

(i) $J\left(a \delta_{l} \phi, a \delta_{l} \psi\right)=J(\phi, \psi)$;

(ii) $J(|\phi|,|\psi|) \leq J(\phi, \psi)$;

(iii) $J^{\prime}\left(a \delta_{l} \phi, a \delta_{l} \psi\right)(u, v)=a^{-1} J^{\prime}(\phi, \psi)\left(\delta_{l^{-1}} u, \delta_{l^{-1}} v\right)$.

In addition, if $\phi$ and $\psi$ are non-negative then

(iv) $J\left(\phi^{*}, \psi^{*}\right) \leq J(\phi, \psi)$.

Proof. The proofs are immediate consequences of Lemma 2.8 .

Now we are able to proof Theorem 2.5. We will follow the arguments presented in references [9] and [22].

Proof of Theorem [2.5. Let $\left\{\left(\phi_{j}, \psi_{j}\right)\right\} \subset \mathcal{P}$ be a minimizing sequence for $J$, i.e.,

$$
\lim _{j \rightarrow \infty} J\left(\phi_{j}, \psi_{j}\right)=\alpha_{1} .
$$

In view of Lemma 2.9 we have $J\left(\left|\phi_{j}\right|,\left|\psi_{j}\right|\right) \leq J\left(\phi_{j}, \psi_{j}\right)$. So, we may assume that $\phi_{j}, \psi_{j}$ are non-negative. In addition, also from Lemma 2.9, $J\left(\phi_{j}^{*}, \psi_{j}^{*}\right) \leq J\left(\phi_{j}, \psi_{j}\right)$; thus, we also may assume that $\phi_{j}, \psi_{j}$ are radially symmetric and nonincreasing functions in $H^{1}$. Define

$$
\tilde{\phi}_{j}=t_{j} \delta_{l_{j}} \phi_{j} \quad \text { and } \quad \tilde{\psi}_{j}=t_{j} \delta_{l_{j}} \psi_{j}
$$

where

$$
t_{j}=\frac{Q\left(\phi_{j}, \psi_{j}\right)^{\frac{n}{4}-\frac{1}{2}}}{K\left(\phi_{j}, \psi_{j}\right)^{\frac{n}{4}}} \quad \text { and } \quad l_{j}=\frac{K\left(\phi_{j}, \psi_{j}\right)^{\frac{1}{2}}}{Q\left(\phi_{j}, \psi_{j}\right)^{\frac{1}{2}}} .
$$

An application of Lemma 2.8, with $a=t_{j}$ and $l=l_{j}$, gives

$$
K\left(\tilde{\phi}_{j}, \tilde{\psi}_{j}\right)=Q\left(\tilde{\phi}_{j}, \tilde{\psi}_{j}\right)=1
$$

Hence,

$$
\frac{1}{P\left(\tilde{\phi}_{j}, \tilde{\psi}_{j}\right)}=\frac{Q\left(\tilde{\phi}_{j}, \tilde{\psi}_{j}\right)^{\frac{3}{2}-\frac{n}{4}} K\left(\tilde{\phi}_{j}, \tilde{\psi}_{j}\right)^{\frac{n}{4}}}{P\left(\tilde{\phi}_{j}, \tilde{\psi}_{j}\right)}=J\left(\tilde{\phi}_{j}, \tilde{\psi}_{j}\right)=J\left(\phi_{j}, \psi_{j}\right) \rightarrow \alpha_{1}>0 .
$$

Recall that $H_{r d}^{1}\left(\mathbb{R}^{n}\right)$ denotes the closed subspace of $H^{1}\left(\mathbb{R}^{n}\right)$ composed by radially symmetric and nonincreasing functions. It follows from (2.10) that sequence $\left(\tilde{\phi}_{j}, \tilde{\psi}_{j}\right)$ is bounded in the 
space $H_{r d}^{1}\left(\mathbb{R}^{n}\right) \times H_{r d}^{1}\left(\mathbb{R}^{n}\right)$. Consequently, there exist a subsequence, still denoted by $\left(\tilde{\phi}_{j}, \tilde{\psi}_{j}\right)$, and functions $\phi_{0}, \psi_{0} \in H_{r d}^{1}\left(\mathbb{R}^{n}\right)$ such that

$$
\tilde{\phi}_{j} \rightarrow \phi_{0}, \quad \tilde{\psi}_{j} \rightarrow \psi_{0}, \quad \text { in } \quad H^{1}\left(\mathbb{R}^{n}\right) .
$$

By recalling the compactness of the embedding $H_{r d}^{1}\left(\mathbb{R}^{n}\right) \hookrightarrow L^{3}\left(\mathbb{R}^{n}\right), 1 \leq n \leq 5$, we see that $\left(\tilde{\phi}_{j}, \tilde{\psi}_{j}\right) \rightarrow\left(\phi_{0}, \psi_{0}\right)$, in $L^{3} \times L^{3}$ and almost everywhere. This immediately implies that $\phi_{0}$ and $\psi_{0}$ are non-negative and

$$
\lim _{j \rightarrow \infty} P\left(\tilde{\phi}_{j}, \tilde{\psi}_{j}\right)=P\left(\phi_{0}, \psi_{0}\right)
$$

Therefore by (2.11) we get

$$
P\left(\phi_{0}, \psi_{0}\right)=\lim _{j \rightarrow \infty} P\left(\tilde{\phi}_{j}, \tilde{\psi}_{j}\right)=\alpha_{1}^{-1}>0,
$$

which means that $\left(\phi_{0}, \psi_{0}\right) \in \mathcal{P}$.

On the other hand, the lower semi-continuity of the weak convergence gives

$$
Q\left(\phi_{0}, \psi_{0}\right) \leq \liminf _{j \rightarrow \infty} Q\left(\tilde{\phi}_{j}, \tilde{\psi}_{j}\right)=1
$$

and

$$
K\left(\phi_{0}, \psi_{0}\right) \leq \liminf _{j \rightarrow \infty} K\left(\tilde{\phi}_{j}, \tilde{\psi}_{j}\right)=1 .
$$

Therefore, the definitions of $\alpha_{1}$ and $J$ and (2.12) yield

$$
\alpha_{1} \leq J\left(\phi_{0}, \psi_{0}\right)=\frac{Q\left(\phi_{0}, \psi_{0}\right)^{\frac{3}{2}-\frac{n}{4}} K\left(\phi_{0}, \psi_{0}\right)^{\frac{n}{4}}}{P\left(\phi_{0}, \psi_{0}\right)} \leq \frac{1}{P\left(\phi_{0}, \psi_{0}\right)}=\alpha_{1} .
$$

From (2.13) we then conclude that

$$
J\left(\phi_{0}, \psi_{0}\right)=\alpha_{1}
$$

and

$$
K\left(\phi_{0}, \psi_{0}\right)=Q\left(\phi_{0}, \psi_{0}\right)=1 .
$$

This last assertion also implies that $\tilde{\phi}_{j} \rightarrow \phi_{0}, \tilde{\psi}_{j} \rightarrow \psi_{0}$ strongly in $H^{1}$. Part (i) of the theorem is thus established.

For part (ii), we start by noting that for any $(u, v) \in H^{1} \times H^{1}$ and $t$ sufficiently small, $\left(\phi_{0}+t u, \psi_{0}+t v\right) \in \mathcal{P}$. Thus, since $\left(\phi_{0}, \psi_{0}\right)$ is a minimizer of $J$ on $\mathcal{P}$,

$$
\left.\frac{d}{d t}\right|_{t=0} J\left(\phi_{0}+t u, \psi_{0}+t v\right)=0 .
$$

Using Lemma 2.8 this is equivalent to

$$
\begin{array}{r}
\frac{Q\left(\phi_{0}, \psi_{0}\right)^{\frac{3}{2}-\frac{n}{4}} K\left(\phi_{0}, \psi_{0}\right)^{\frac{n}{4}}}{P\left(\phi_{0}, \psi_{0}\right)}\left[\frac{n}{4} \frac{K^{\prime}\left(\phi_{0}, \psi_{0}\right)(u, v)}{K\left(\phi_{0}, \psi_{0}\right)}+\left(\frac{3}{2}-\frac{n}{4}\right) \frac{1}{Q\left(\phi_{0}, \psi_{0}\right)} Q^{\prime}\left(\phi_{0}, \psi_{0}\right)(u, v)\right] \\
=\frac{Q\left(\phi_{0}, \psi_{0}\right)^{\frac{3}{2}-\frac{n}{4}} K\left(\phi_{0}, \psi_{0}\right)^{\frac{n}{4}}}{P\left(\phi_{0}, \psi_{0}\right)^{2}} P^{\prime}\left(\phi_{0}, \psi_{0}\right)(u, v) .
\end{array}
$$

In view of (2.12) and (2.15), this yields, for any $(u, v) \in H^{1} \times H^{1}$,

$$
K^{\prime}\left(\phi_{0}, \psi_{0}\right)(u, v)+\frac{6-n}{n} Q^{\prime}\left(\phi_{0}, \psi_{0}\right)(u, v)=\frac{4 \alpha_{1}}{n} P^{\prime}\left(\phi_{0}, \psi_{0}\right)(u, v) .
$$

Next, define $(\phi, \psi)=\left(t_{0} \delta_{l_{0}} \phi_{0}, t_{0} \delta_{l_{0}} \psi_{0}\right)$ with

$$
t_{0}=\frac{2 \alpha_{1}}{6-n} \quad \text { and } \quad l_{0}=\left(\frac{6-n}{n}\right)^{1 / 2} \text {. }
$$


We claim that $(\phi, \psi)$ is a solution of (2.1) with $\omega=1$, that is, $(\phi, \psi)$ is a critical point of $I_{1}$. To see this, we note that for any $u, v \in H^{1}$, in view of Lemma 2.8 ,

$$
\begin{aligned}
& I_{1}^{\prime}(\phi, \psi)(u, v) \\
& =\frac{1}{2}\left[K^{\prime}\left(t_{0} \delta_{l_{0}} \phi_{0}, t_{0} \delta_{l_{0}} \psi_{0}\right)(u, v)+Q^{\prime}\left(t_{0} \delta_{l_{0}} \phi_{0}, t_{0} \delta_{l_{0}} \psi_{0}\right)(u, v)\right]-P^{\prime}\left(t_{0} \delta_{l_{0}} \phi_{0}, t_{0} \delta_{l_{0}} \psi_{0}\right)(u, v) \\
& =\frac{t_{0} l_{0}^{n-2}}{2}\left[K^{\prime}\left(\phi_{0}, \psi_{0}\right)\left(\delta_{l_{0}^{-1}} u, \delta_{l_{0}^{-1}} v\right)+l_{0}^{2} Q^{\prime}\left(\phi_{0}, \psi_{0}\right)\left(\delta_{l_{0}^{-1}} u, \delta_{l_{0}^{-1}} v\right)-2 t_{0} l_{0}^{2} P^{\prime}\left(\phi_{0}, \psi_{0}\right)\left(\delta_{l_{0}^{-1}} u, \delta_{l_{0}^{-1}} v\right)\right] \\
& =\frac{t_{0} l_{0}^{n-2}}{2}\left[K^{\prime}\left(\phi_{0}, \psi_{0}\right)\left(\delta_{l_{0}^{-1}} u, \delta_{l_{0}^{-1}} v\right)+\frac{6-n}{n} Q^{\prime}\left(\phi_{0}, \psi_{0}\right)\left(\delta_{l_{0}^{-1}} u, \delta_{l_{0}^{-1}} v\right)\right. \\
& \left.\quad-\frac{4 \alpha_{1}}{n} P^{\prime}\left(\phi_{0}, \psi_{0}\right)\left(\delta_{l_{0}^{-1}} u, \delta_{l_{0}^{-1}} v\right)\right] \\
& =0,
\end{aligned}
$$

where in the last line we used (2.16).

Now from Lemmas 2.3 and 2.9 we have that $(\phi, \psi)$ is also a critical point of $J$ with $J(\phi, \psi)=$ $J\left(\phi_{0}, \psi_{0}\right)$. Since $\left(\phi_{0}, \psi_{0}\right)$ is a minimizer of $J$, so is $(\phi, \psi)$. An application of Corollary 2.4 then gives that $(\phi, \psi)$ is a ground state of (2.1) with $\omega=1$. Finally, the positiveness of $(\phi, \psi)$ follows from the maximum principle. This shows part (ii).

Next we will prove the relation (2.9). Indeed, if $(\phi, \psi)$ is as in part (ii), Lemmas 2.3 and 2.1 imply,

$$
\begin{aligned}
\alpha_{1} & =J(\phi, \psi) \\
& =\frac{n^{\frac{n}{4}}}{2}(6-n)^{\frac{3}{2}-\frac{n}{4}} I_{1}(\phi, \psi)^{\frac{1}{2}} \\
& =\frac{n^{\frac{n}{4}}}{2}(6-n)^{1-\frac{n}{4}} Q(\phi, \psi)^{\frac{1}{2}} .
\end{aligned}
$$

Therefore, if $(\tilde{\phi}, \tilde{\psi})$ is any ground state of (2.1), with $\omega=1$, Remark 2.2 yields

$$
\alpha_{1}=\frac{n^{\frac{n}{4}}}{2}(6-n)^{1-\frac{n}{4}} Q(\tilde{\phi}, \tilde{\psi})^{\frac{1}{2}} .
$$

The proof of the theorem is thus completed.

From the proof of Lemma 2.7 we deduce the existence of a constant $C>0$ such that the Gagliardo-Nirenberg-type inequality

$$
P(u, v) \leq C Q(u, v)^{\frac{3}{2}-\frac{n}{4}} K(u, v)^{\frac{n}{4}}
$$

holds, for any $(u, v) \in \mathcal{P}$. In view of Theorem 2.5 the sharp constant one can place in (2.17) is $\alpha_{1}^{-1}$. More precisely, we have.

Corollary 2.10. Let $1 \leq n \leq 5$. Then the inequality

$$
P(u, v) \leq C_{o p} Q(u, v)^{\frac{3}{2}-\frac{n}{4}} K(u, v)^{\frac{n}{4}}
$$

holds, for any $(u, v) \in \mathcal{P}$, with

$$
C_{o p}=\frac{2(6-n)^{\frac{n}{4}-1}}{n^{\frac{n}{4}}} \frac{1}{Q(\phi, \psi)^{\frac{1}{2}}},
$$

where $(\phi, \psi)$ is any ground state solution of (2.1) with $\omega=1$.

Remark 2.11. When $n=4$, we recover the best constant obtained by the authors in [9, Theorem 5.1]. 
2.3. Characterization of ground states. Next we present a characterization of the minimizers of $J$. The result generalizes the one for $n=4$ present in [9, Theorem 5.1].

Theorem 2.12. Let $\alpha_{1}$ be defined as in (2.8). Then the set of minimizers of $J$ is characterized as

$$
\left\{(\phi, \psi) \in H^{1} \times H^{1} ; J(\phi, \psi)=\alpha_{1}\right\}=\left\{\left(t \delta_{l} \phi, t \delta_{l} \psi\right) \in H^{1} \times H^{1} ; t, l>0,(\phi, \psi) \in \mathcal{G}_{1}\right\} .
$$

Proof. Consider the following sets

$$
A=\left\{(\phi, \psi) \in H^{1} \times H^{1} ; J(\phi, \psi)=\alpha_{1}\right\}
$$

and

$$
B=\left\{\left(t \delta_{l} \phi, t \delta_{l} \psi\right) \in H^{1} \times H^{1} ; t, l>0,(\phi, \psi) \in \mathcal{G}_{1}\right\} .
$$

If $\left(\phi_{0}, \psi_{0}\right) \in A$ then $J\left(\phi_{0}, \psi_{0}\right)=\alpha_{1}$ and from Theorem 2.5 there exist $t_{0}, l_{0}>0$ such that $(\phi, \psi)=\left(t_{0} \delta_{l_{0}} \phi_{0}, t_{0} \delta_{l_{0}} \psi_{0}\right)$ is a ground state of (2.1). This means that

$$
\left(\phi_{0}, \psi_{0}\right)=\left(\frac{1}{t_{0}} \delta_{l_{0}^{-1}} \phi, \frac{1}{t_{0}} \delta_{l_{0}^{-1}} \psi\right)
$$

with $(\phi, \psi) \in \mathcal{G}_{1}$ or, equivalently, $\left(\phi_{0}, \psi_{0}\right) \in B$.

On the other hand, assume $\left(\phi_{0}, \psi_{0}\right) \in B$, that is, $\left(\phi_{0}, \psi_{0}\right)=\left(t \delta_{l} \phi, t \delta_{l} \psi\right)$ for some $t, l>0$ with $(\phi, \psi) \in \mathcal{G}_{1}$. We must proof that $J\left(\phi_{0}, \psi_{0}\right)=\alpha_{1}$. From Lemma 2.9 we have $J\left(\phi_{0}, \psi_{0}\right)=$ $J(\phi, \psi)$. But since $(\phi, \psi) \in \mathcal{G}_{1}$, Corollary 2.4 implies that $(\phi, \psi)$ is a minimizer of $J$, that is, $J(\phi, \psi)=\alpha_{1}$. Consequently, $J\left(\phi_{0}, \psi_{0}\right)=\alpha_{1}$ and $\left(\phi_{0}, \psi_{0}\right) \in A$.

\section{Global existence versus Blow up}

In this section, we prove Theorems 1.1 and 1.2. Before proving the results, we need some previous tools. We start with two lemmas. Their proofs can be found, for instance, in references [1], [5], and [18].

Lemma 3.1. Let $I$ be an open interval with $0 \in I$. Let $a \in \mathbb{R}$ and $b>0$. Define $\gamma=(b q)^{-\frac{1}{q-1}}$ and $f(r)=a-r+b r^{q}$, for $r \geq 0$. Let $G(t)$ be a nonnegative continuous function such that $f \circ G \geq 0$ on $I$. Assume that $a<\left(1-\frac{1}{q}\right) \gamma$.

(i) If $G(0)<\gamma$, then $G(t)<\gamma, \forall t \in I$.

(ii) If $G(0)>\gamma$, then $G(t)>\gamma, \forall t \in I$.

Corollary 3.2. Let $I$ be an open interval with $0 \in I$. Let $a \in \mathbb{R}$ and $b>0$. Define $\gamma=(b q)^{-\frac{1}{q-1}}$ and $f(r)=a-r+b r^{q}$, for $r \geq 0$. Let $G(t)$ be a nonnegative continuous function such that $f \circ G \geq 0$ on $I$. Assume that $a<\left(1-\delta_{1}\right)\left(1-\frac{1}{q}\right) \gamma$, for some small $\delta_{1}>0$. If $G(0)>\gamma$, then there exists $\delta_{2}=\delta_{2}\left(\delta_{1}\right)>0$ such that $G(t)>\left(1+\delta_{2}\right) \gamma, \forall t \in I$.

3.1. Global existence in $H^{1}\left(\mathbb{R}^{5}\right)$. By using Corollary 2.10 and standard arguments one can show if $\left\|\left(u_{0}, v_{0}\right)\right\|_{H^{1} \times H^{1}} \leq \rho$, for some $\rho$ sufficiently small then the corresponding solution of (1.4) is global in $H^{1}\left(\mathbb{R}^{5}\right) \times H^{1}\left(\mathbb{R}^{5}\right)$ (see, for instance, [16]). Particularly, if $\rho$ is small then the assumptions of Theorem 1.1 hold. Thus, Theorem 1.1 can be seen as an answer to the question of how small $\rho$ must be.

The proof of Theorem 1.1 will follow as an application of Lemma 3.1. To simplify it we first prove the following.

Lemma 3.3. Let $(u(t), v(t))$ be the solution of (1.4) with initial data $\left(u_{0}, v_{0}\right) \in H^{1}\left(\mathbb{R}^{5}\right) \times$ $H^{1}\left(\mathbb{R}^{5}\right)$. Define $G(t)=K(u(t), v(t)), a=E\left(u_{0}, v_{0}\right), b=2 C_{o p} Q\left(u_{0}, v_{0}\right)^{\frac{1}{4}}$ and $q=\frac{5}{4}$. Then,

(i) $f \circ G \geq 0$, where $f(r)=a-r+b r^{q}$.

(ii) If $\gamma=(b q)^{-\frac{1}{q-1}}$ then 

(a) $a<\left(1-\frac{1}{q}\right) \gamma \Longleftrightarrow E\left(u_{0}, v_{0}\right) Q\left(u_{0}, v_{0}\right)<E(\phi, \psi) Q(\phi, \psi)$;
(b) $G(0)<\gamma \Longleftrightarrow Q\left(u_{0}, v_{0}\right) K\left(u_{0}, v_{0}\right)<Q(\phi, \psi) K(\phi, \psi)$.

Proof. For part (i), from the definition of the energy and Corollary 2.10, with $n=5$, we have

$$
\begin{aligned}
K(u(t), v(t)) & =E\left(u_{0}, v_{0}\right)+2 \operatorname{Re}\left(v(t), u(t)^{2}\right)_{L^{2}} \\
& \leq E\left(u_{0}, v_{0}\right)+2 P\left(|u(t)|^{2},|v(t)|\right) \\
& \leq E\left(u_{0}, v_{0}\right)+2 C_{o p} Q(u(t), v(t))^{\frac{1}{4}} K(u(t), v(t))^{\frac{5}{4}} .
\end{aligned}
$$

The conservation of the charge then gives part (i).

For part (ii), we first observe that from Lemma 2.1 with $n=5$ and $\omega=1$,

$$
Q(\phi, \psi)=\frac{1}{5} K(\phi, \psi) \quad \text { and } \quad P(\phi, \psi)=2 Q(\phi, \psi) .
$$

Hence,

$$
E(\phi, \psi)=K(\phi, \psi)-2 P(\phi, \psi)=5 Q(\phi, \psi)-4 Q(\phi, \psi)=Q(\phi, \psi) .
$$

In addition, by definition,

$$
\gamma=5 \frac{Q(\phi, \psi)^{2}}{Q\left(u_{0}, v_{0}\right)} .
$$

By combining (3.1)-(3.2), part (ii) follows from straightforward calculations.

Finally we are able to prove Theorem 1.1.

Proof of Theorem 1.1. From Lemma 3.3. assumptions (1.8) and (1.9) are equivalent to $a<$ $\left(1-\frac{1}{q}\right) \gamma$ and $G(0)<\gamma$. Thus from Lemma 3.1 we conclude that $G(t)<\gamma$, or equivalently,

$$
Q\left(u_{0}, v_{0}\right) K(u(t), v(t))<Q(\phi, \psi) K(\phi, \psi), \quad \forall t \in I .
$$

This combined with the conservation of the charge implies an a priori estimate for the solution in $H^{1} \times H^{1}$. Consequently, the solution can be extended globally-in-time and the proof is completed.

3.2. Existence of blow-up solutions. Here we will show Theorem 1.2, Before starting with the proof itself, we need some preliminary results. First we recall the virial identities for system (1.4). The first one is used to prove the blow up under the assumption of finite variance.

Lemma 3.4. Assume $1 \leq n \leq 6$ and $\kappa=1 / 2$. Let $(u(t), v(t))$ be the unique solution of (1.4) with $\left(u_{0}, v_{0}\right) \in H^{1}\left(\mathbb{R}^{n}\right) \times H^{1}\left(\mathbb{R}^{n}\right)$ and $\left(x u_{0}, x v_{0}\right) \in L^{2}\left(\mathbb{R}^{n}\right) \times L^{2}\left(\mathbb{R}^{n}\right)$. Then, as long as the solution exists,

$$
\frac{d^{2}}{d t^{2}} Q(x u(t), x v(t))=2 n E\left(u_{0}, v_{0}\right)+2(4-n) K(u(t), v(t)) .
$$

Proof. See [9, Theorem 3.11]. In particular, in [9, Theorem 3.8] it was shown that, under our assumptions, the local solution also satisfies $(x u(t), x v(t)) \in L^{2}\left(\mathbb{R}^{n}\right) \times L^{2}\left(\mathbb{R}^{n}\right)$.

Theorem 3.5. Assume $1 \leq n \leq 6$ and $\kappa=1 / 2$. Let $(u(t), v(t))$ be the unique solution of (1.4) with $\left(u_{0}, v_{0}\right) \in H^{1}\left(\mathbb{R}^{n}\right) \times H^{1}\left(\mathbb{R}^{n}\right)$. Let $\varphi \in C_{0}^{\infty}\left(\mathbb{R}^{n}\right)$ and define

$$
V(t)=\frac{1}{2} \int \varphi(x)\left(|u|^{2}+2|v|^{2}\right) d x .
$$

Then,

$$
V^{\prime}(t)=\operatorname{Im} \int \nabla \varphi \cdot \nabla u \bar{u} d x+\operatorname{Im} \int \nabla \varphi \cdot \nabla v \bar{v} d x
$$


and

$$
\begin{aligned}
V^{\prime \prime}(t)= & 2 \sum_{1 \leq k, j \leq n} \operatorname{Re} \int \frac{\partial^{2} \varphi}{\partial x_{k} \partial x_{j}}\left[\partial x_{j} \bar{u} \partial x_{k} u+\frac{1}{2} \partial x_{j} \bar{v} \partial x_{k} v\right] d x \\
& -\frac{1}{2} \int \Delta^{2} \varphi\left(|u|^{2}+\frac{1}{2}|v|^{2}\right) d x-\operatorname{Re} \int \Delta \varphi \bar{v} u^{2} d x
\end{aligned}
$$

Proof. The proof follows the ideas presented in Lemma 2.9 of [17], where the virial identity was established for the classical Schrödinger equation. So we omit the details. An adapted version for Schrödinger-type systems, can also be found in [18].

Corollary 3.6. Under the assumptions of Theorem 3.5, if $u, v$ and $\varphi$ are radially symmetric functions then we can write 3.4 as

$$
\begin{aligned}
V^{\prime \prime}(t)= & 2 \int \varphi^{\prime \prime}\left(|\nabla u|^{2}+\frac{1}{2}|\nabla v|^{2}\right) d x-\frac{1}{2} \int \Delta^{2} \varphi\left(|u|^{2}+\frac{1}{2}|v|^{2}\right) d x \\
& -\operatorname{Re} \int \Delta \varphi \bar{v} u^{2} d x
\end{aligned}
$$

Proof. The proof follows immediately from Theorem 3.5.

We will use Corollary 3.6 with $\varphi$ replaced by the function $\chi_{R}$ given in next lemma.

Lemma 3.7. Let $r=|x|, x \in \mathbb{R}^{n}$. Define

$$
\chi(r)=\left\{\begin{array}{cc}
r^{2}, & 0 \leq r \leq 1, \\
0, & r \geq 3,
\end{array}\right.
$$

with $\chi^{\prime \prime}(r) \leq 2$, for any $r \geq 0$. For $R>0$, let $\chi_{R}(r)=R^{2} \chi(r / R)$.

(i) If $r \leq R$, then $\Delta \chi_{R}(r)=2 n$ and $\Delta^{2} \chi_{R}(r)=0$;

(ii) If $r \geq R$, then

$$
\Delta \chi_{R}(r) \leq C_{1}, \quad \Delta^{2} \chi_{R}(r) \leq \frac{C_{2}}{R^{2}}
$$

where $C_{1}, C_{2}$ are constant depending only on $n$.

Proof. The lemma follows by a straightforward calculation.

Finally, we recall a truncated version of the Gagliardo-Nirenberg inequality.

Lemma 3.8. If $u \in H^{1}\left(\mathbb{R}^{5}\right)$ is a radially symmetric function, then

$$
\|u\|_{L^{3}(|x| \geq R)}^{3} \leq \frac{C}{R^{2}}\|u\|_{L^{2}(|x| \geq R)}^{5 / 2}\|\nabla u\|_{L^{2}(|x| \geq R)}^{1 / 2}
$$

Proof. The proof is a consequence of Strauss' radial Lemma (See also [19, page 323]).

Finally, we are in a position to prove the existence of blow-up solutions. We will follow the strategy presented in [11, [18] and [19].

Proof of Theorem 1.2. Since we are assuming (1.10) we have the existence of $\delta_{1}>0$ such that

$$
E\left(u_{0}, v_{0}\right) Q\left(u_{0}, v_{0}\right)<\left(1-\delta_{1}\right) E(\phi, \psi) Q(\phi, \psi)
$$

Hence, as in the proof of Lemma 3.3, we are in the assumptions of Corollary 3.2. Consequently, there exists $\delta_{2}>0$ such that

$$
Q\left(u_{0}, v_{0}\right) K(u(t), v(t))>\left(1+\delta_{2}\right) Q(\phi, \psi) K(\phi, \psi) .
$$


We first assume that $\left(x u_{0}, x v_{0}\right) \in L^{2}\left(\mathbb{R}^{5}\right) \times L^{2}\left(\mathbb{R}^{5}\right)$. Then multiplying both side of (3.3) by $Q\left(u_{0}, v_{0}\right)$ we have

$$
\begin{aligned}
{\left[\frac{d^{2}}{d t^{2}} Q(x u, x v)\right] Q\left(u_{0}, v_{0}\right) } & =10 E\left(u_{0}, v_{0}\right) Q\left(u_{0}, v_{0}\right)-2 K(u, v) Q\left(u_{0}, v_{0}\right) \\
& <10\left(1-\delta_{1}\right) E(\phi, \psi) Q(\phi, \psi)-2 K(u, v) Q\left(u_{0}, v_{0}\right) \\
& <10\left(1-\delta_{1}\right) E(\phi, \psi) Q(\phi, \psi)-2\left(1+\delta_{2}\right) Q(\phi, \psi) K(\phi, \psi) \\
& =2\left(1-\delta_{1}\right) K(\phi, \psi) Q(\phi, \psi)-2\left(1+\delta_{2}\right) Q(\phi, \psi) K(\phi, \psi) \\
& =-2\left(\delta_{1}+\delta_{2}\right) Q(\phi, \psi) K(\phi, \psi)=-B,
\end{aligned}
$$

where we have used that $E(\phi, \psi)=K(\phi, \psi) / 5$. Since $B$ is a positive constant, by using standard arguments, the last inequality is enough to show that $I$ must be finite.

Next, we assume that $u_{0}, v_{0}$ are radial functions. Because the linear and nonlinear parts in (1.4) are invariant by rotations, it is easy to see that $u(t), v(t)$ are also radial functions.

Let $\chi_{R}$ be defined as in Lemma 3.7. The parameter $R$ is fixed at this moment but it will be chosen sufficiently large later. By taking $\varphi$ as $\chi_{R}$ in (3.5), we obtain

$$
\begin{aligned}
V^{\prime \prime}(t)= & 2 \int \chi_{R}^{\prime \prime}\left(|\nabla u|^{2}+\frac{1}{2}|\nabla v|^{2}\right) d x-\frac{1}{2} \int \Delta^{2} \chi_{R}\left(|u|^{2}+\frac{1}{2}|v|^{2}\right) d x \\
& -\operatorname{Re} \int \Delta \chi_{R} \bar{v} u^{2} d x .
\end{aligned}
$$

We will estimate each term on the right-hand side of (3.11). For the first one, by using the fact that $\chi_{R}^{\prime \prime}(r) \leq 2$, we have

$$
2 \int \chi_{R}^{\prime \prime}\left(|\nabla u|^{2}+\frac{1}{2}|\nabla v|^{2}\right) d x \leq 4 \int\left(|\nabla v|^{2}+\frac{1}{2}|\nabla u|^{2}\right) d x=4 K(u, v) .
$$

For the second one we use Lemma 3.7 and the conservation of the charge to get

$$
\begin{aligned}
-\frac{1}{2} \int \Delta^{2} \chi_{R}\left(|u|^{2}+\frac{1}{2}|v|^{2}\right) d x & =-\frac{1}{2} \int_{|x| \geq R} \Delta^{2} \chi_{R}\left(|u|^{2}+\frac{1}{2}|v|^{2}\right) d x \\
& \leq \frac{C_{2}}{R^{2}} \int_{|x| \geq R}\left(|u|^{2}+\frac{1}{2}|v|^{2}\right) d x \\
& \leq \frac{C_{2}^{\prime}}{R^{2}} \int_{\mathbb{R}^{5}}\left(|u|^{2}+2|v|^{2}\right) d x \\
& =\frac{C_{2}^{\prime}}{R^{2}} Q\left(u_{0}, v_{0}\right),
\end{aligned}
$$

where $C_{2}^{\prime}$ is a positive constant.

Finally, in view of Lemma 3.7 the last term in (3.11) is estimated by

$$
\begin{aligned}
-\operatorname{Re} \int \Delta \chi_{R} \bar{v} u^{2} d x & =-\operatorname{Re} \int_{|x| \leq R} \Delta \chi_{R} \bar{v} u^{2} d x-\operatorname{Re} \int_{|x| \geq R} \Delta \chi_{R} \bar{v} u^{2} d x \\
& \leq-10 \operatorname{Re} \int_{|x| \leq R} \bar{v} u^{2} d x+C_{1} \int_{|x| \geq R}|v||u|^{2} d x \\
& \leq-10 \operatorname{Re} \int_{\mathbb{R}^{5}} \bar{v} u^{2} d x+C_{1}^{\prime} \int_{|x| \geq R}|v||u|^{2} d x \\
& =5 E(u, v)-5 K(u, v)+C_{1}^{\prime} \int_{|x| \geq R}|v||u|^{2} d x .
\end{aligned}
$$


where $C_{1}^{\prime}$ is also a positive constant. Gathering together (3.12), (3.13) and (3.14) and using the conservation of the energy we get

$$
V^{\prime \prime}(t) \leq 5 E\left(u_{0}, v_{0}\right)-K(u, v)+\frac{C_{2}^{\prime}}{R^{2}} Q\left(u_{0}, v_{0}\right)+C_{1}^{\prime} \int_{|x| \geq R}|v||u|^{2} d x .
$$

Now, for the last integral in (3.15) we use Young's inequality to obtain

$$
C_{1}^{\prime} \int_{|x| \geq R}|v||u|^{2} d x \leq \frac{2 C_{1}^{\prime}}{3} \int_{|x| \geq R}|u|^{3} d x+\frac{C_{1}^{\prime}}{3} \int_{|x| \geq R}|v|^{3} d x .
$$

Then using Lemma 3.8 and Young's inequality with $\epsilon$, we deduce that

$$
\begin{aligned}
C_{1}^{\prime} \int_{|x| \geq R}\left|v\left\|\left.u\right|^{2} d x \leq \frac{C}{R^{2}}\right\| u\left\|_{L^{2}(|x| \geq R)}^{5 / 2}\right\| \nabla u\left\|_{\left.L^{2}|x| \geq R\right)}^{1 / 2}+\frac{C}{R^{2}}\right\| v\left\|_{L^{2}(|x| \geq R)}^{5 / 2}\right\| \nabla v \|_{\left.L^{2}|x| \geq R\right)}^{1 / 2}\right. \\
\leq \frac{C_{\epsilon}}{R^{8 / 3}}\left(\|u\|_{L^{2}(|x| \geq R)}^{10 / 3}+\|v\|_{L^{2}(|x| \geq R)}^{10 / 3}\right)+\epsilon\left(\|\nabla u\|_{L^{2}(|x| \geq R)}^{2}+\frac{1}{2}\|\nabla v\|_{L^{2}(|x| \geq R)}^{2}\right) \\
\leq \frac{\tilde{C}_{\epsilon}}{R^{8 / 3}}\left(\|u\|_{L^{2}\left(\mathbb{R}^{5}\right)}^{2}+2\|v\|_{L^{2}\left(\mathbb{R}^{5}\right)}^{2}\right)^{5 / 3}+\epsilon\left(\|\nabla u\|_{L^{2}\left(\mathbb{R}^{5}\right)}^{2}+\frac{1}{2}\|\nabla v\|_{L^{2}\left(\mathbb{R}^{5}\right)}^{2}\right) \\
\quad=\frac{\tilde{C}_{\epsilon}}{R^{8 / 3}} Q\left(u_{0}, v_{0}\right)^{5 / 3}+\epsilon K(u, v) .
\end{aligned}
$$

Therefore, replacing (3.16) into (3.15), we conclude

$$
V^{\prime \prime}(t) \leq 5 E\left(u_{0}, v_{0}\right)-(1-\epsilon) K(u, v)+\frac{C_{2}}{R^{2}} Q\left(u_{0}, v_{0}\right)+\frac{\tilde{C}_{\epsilon}}{R^{8 / 3}} Q\left(u_{0}, v_{0}\right)^{5 / 3} .
$$

Multiplying (3.17) by $Q\left(u_{0}, v_{0}\right)$, we infer

$$
\begin{aligned}
Q\left(u_{0}, v_{0}\right) V^{\prime \prime}(t) \leq & 5 E\left(u_{0}, v_{0}\right) Q\left(u_{0}, v_{0}\right)-(1-\epsilon) K(u, v) Q\left(u_{0}, v_{0}\right) \\
& +\frac{C_{2}}{R^{2}} Q\left(u_{0}, v_{0}\right)^{2}+\frac{\tilde{C}_{\epsilon}}{R^{8 / 3}} Q\left(u_{0}, v_{0}\right)^{8 / 3}
\end{aligned}
$$

Using (3.9), (3.10) and the fact that $E(\phi, \psi)=K(\phi, \psi) / 5$, we finally obtain

$$
\begin{aligned}
Q\left(u_{0}, v_{0}\right) V^{\prime \prime}(t) \leq & \left(1-\delta_{1}\right) Q(\phi, \psi) K(\phi, \psi)-(1-\epsilon)\left(1+\delta_{2}\right) Q(\phi, \psi) K(\phi, \psi) \\
& +\frac{C_{2}}{R^{2}} Q\left(u_{0}, v_{0}\right)^{2}+\frac{\tilde{C}_{\epsilon}}{R^{8 / 3}} Q\left(u_{0}, v_{0}\right)^{8 / 3} \\
= & {\left[-\delta_{1}-\delta_{2}+\epsilon\left(1+\delta_{2}\right)\right] Q(\phi, \psi) K(\phi, \psi)+\frac{C_{2}}{R^{2}} Q\left(u_{0}, v_{0}\right)^{2}+\frac{\tilde{C}_{\epsilon}}{R^{8 / 3}} Q\left(u_{0}, v_{0}\right)^{8 / 3} . }
\end{aligned}
$$

By choosing $\epsilon>0$ small enough and $R>0$ large enough, we conclude that $V^{\prime \prime}(t)<-B$, for some $B>0$. As before, this is enough to show that $I$ must be finite.

Remark 3.9. If $E\left(u_{0}, v_{0}\right)<0$, then an application of Corollary 2.10 combined with the fact that $Q(\phi, \psi)=K(\phi, \psi) / 5$ immediately gives

$$
Q\left(u_{0}, v_{0}\right) K\left(u_{0}, v_{0}\right)>\left(\frac{5}{4}\right)^{4} Q(\phi, \psi) K(\phi, \psi),
$$

which in turn shows that (1.11) holds. This is in agreement with the results in [9] where the blow up was shown if the initial energy is negative (see also [4]). However, since $(5 / 4)^{4}>1$, our result is stronger than the one [9]. 


\section{ACKNOWLEDGEMENT}

N.N is supported by a partial scholarship from OAICE (University of Costa Rica). A.P. is partially supported by CNPq/Brazil grants 402849/2016-7 and 303098/2016-3.

\section{REFERENCES}

[1] P. Bégout, Necessary conditions and sufficient conditions for global existence in the nonlinear Schrödinger equation, Adv. Math. Sci. Appl. 12 (2002), 817âĂŞ827.

[2] Th. Cazenave,Semilinear Schrödinger Equations, Courant Lectures Notes in Mathematics, Vol. 10, American Mathematical Society, Providence, 2003.

[3] M. Colin, L. Di Menza, and J.-C. Saut, Solitons in quadratic media, Nonlinearity 29 (2016), 661-690.

[4] A. Corcho, S. Correia, F. Oliveira, and J.D. Silva, On a nonlinear Schrödinger system arising in quadratic media, arXiv 1703.10509v2.

[5] A. Esfahani and A. Pastor, Sharp constant of an anisotropic Gagliardo-Nirenberg-type inequality and applications, Bull. Braz. Math. Soc. 48 (2017), 171-185.

[6] L. Evans, Partial Differential Equations, Graduate Studies in Mathematics, Vol. 19, American Mathematical Society, Second Edition, Providence, 2010.

[7] N. Hayashi, C. Li, and P.I. Naumkin, On a system of nonlinear Schrödinger equations in 2D, Differential Integral Equations 24 (2011), 417-434.

[8] N. Hayashi, C. Li, and T. Ozawa, Small data scattering for a system of nonlinear Schrödinger equations, Differ. Equ. Appl. 3 (2011), 415-426.

[9] N. Hayashi, T. Ozawa, and K. Tanaka, On a system of nonlinear Schrödinger equations with quadratic interaction, Ann. Inst. H. Poincaré Anal. Non Linéaire 30 (2013), 661-690.

[10] J. Holmer and S. Roudenko, On blow-up solutions to the 3D cubic nonlinear Schrödinger equation, Appl. Math. Res. Express. AMRX (2007), Art. ID abm004.

[11] J. Holmer and S. Roudenko, A sharp condition for scattering of the radial 3D cubic nonlinear Schrödinger equation, Commun. Math. Phys. 282 (2008), 435-467.

[12] G. Hoshino and Ozawa, Analytic smoothing effect for a system of Schrödinger equations with two wave interaction, Adv. Differential Equations 20 (2015), 697-716.

[13] G. Leoni, A first course in Sobolev spaces, Graduate Studies in Mathematics, Vol. 105, American Mathematical Society, Providence, 2009.

[14] C. Li, On a system of nonlinear Schrödinger equations and scale invariant spaces in 2D, Differential Integral Equations 28 (2015), 201-220.

[15] Li, C., Hayashi, N., Recent porgress on nonlinear Schrödinger system with quadratic interaction The Scientific World Journal 2014 (2014), Article ID 214821.

[16] F. Linares and G. Ponce, Introduction to Nonlinear Dispersive Equations, Universitex, Springer, New York, 2009.

[17] O. Kavian, A remark on the blowing-up solutions to the Cauchy problem for nonlinear Schrödinger equations, Trans. Am. Math. Soc. 299 (1987), 193-203.

[18] A. Pastor, Weak concentration and wave operator for a 3D coupled nonlinear Schrödinger system, J. Math. Phys. 56 (2015), 021507-1 to 021507-18.

[19] T. Ogawa and Y. Tsutsumi, Blow-up of $H^{1}$ solutions for the nonlinear Schrödinger equation, J. Differential Equations 92 (1991), 317-330.

[20] T. Ozawa and H. Sunagawa, Small data blow-up for a system of nonlinear Schrödinger equations, J. Math. Anal. Appl. 399 (2013), 147-155.

[21] W. Strauss, Existence of solitary waves in higher dimensions, Commun. Math. Phys. 55 (1977), $149-162$.

[22] M. Weinstein, Nonlinear Schrödinger equations and sharp interpolation estimates, Commun. Math. Phys. 87 (1983), 567-576.

IMECC-UniCAMP, Rua Sérgio Buarque de Holanda, 651, 13083-859, Campinas-SP, Brazil E-mail address: nnoguera57@gmail.com

IMECC-UniCAmP, Rua Sérgio Buarque de Holanda, 651, 13083-859, Campinas-SP, Brazil E-mail address: apastor@ime.unicamp.br 